\title{
Evaluation of Side Friction in IHCM for Highway Two Lanes Two Ways
}

\author{
Najid \\ Civil Engineering Department \\ Tarumanegara University \\ Jakarta, Indonesia \\ najid@ ft.untar.ac.id
}

\begin{abstract}
IHCM (Indonesia Highway Capacity Manual) was issued and came into force in 1997. After 20 years there has been a change in traffic from the number and composition, as well as the traffic regulation policy. As a result of this, the determination of IHCM's road capacity is often incorrect. Therefore it is necessary to evaluate IHCM. This study tries to evaluate IHCM through side obstacle factors, as one of the factors that determine the value of road capacity. Research is limited to evaluating the weight of each side friction factor and the effect of land use from the survey results using statistical analysis. From the analysis, it is found that the conditions for the use of shops, markets, hotels, gas stations are in the category of moderate side barriers and the ranking of the relative weights of the side barriers at IHCM is still appropriate.
\end{abstract}

Keywords: traffic volume, side friction, land use

\section{BACKGROUND}

Transportation system is a road network system that physically connects an activity space with other activities space, which influences the development of a region (space of activity) and the development of a space of activity will require an increase in the transportation service system [1].

The development of activity space (activity) mainly occurs on the side of the road. Activities on the side of the road have an impact on traffic performance which can hamper traffic flow, which is then called side friction. Because it influences the flow of traffic it means it also affects the capacity of the road.

Side friction in the IHCM (Indonesian Highway Capacity Manual) are classified consisting of un-motorized vehicles (weight 0.4 ), pedestrians $(0.5)$, left-right access roads (weight 0.7 ) and parked / stopped vehicles (weight 1, 0) [2]. The effect of side friction on capacity is the accumulation of the number of each occurrence of the side resistance factor multiplied by its weight.

In addition to IHCM, 1997 has been too long, the calculation of road capacity based on IHCM is often lower than the volume of traffic observed. Therefore IHCM needs to be evaluated. This research is expected to contribute to the research to revise IHCM in terms of the side effect factors on road capacity.

\section{IDENTIFICATION OF PROBLEMS}

a) Relation of each side friction factor to land use condition. b) The relationship of each side friction factor to traffic volume.

\section{PROBLEM FORMULATION}

From the identification of the above problems can be formulated:

a) What is the relationship between the conditions of land use and the magnitude of each factor in the side friction?

b) How does the traffic condition affected by the value of each side friction factor?

c) How is the difference in the side friction based on IHCM with the survey results?

\section{Purpose ANd OBJECTIVES}

The purpose of this study:

a) Analyze the value of side friction in different land uses.

b) Arrange the correlation between land use with the value of the side friction factor.

c) Evaluate each side friction factor IHCM version

\section{SCOPE OF Problem}

This research is limited by the following scope:

1) Highway configuration $2 / 2$ UD (two lanes two ways undivided)

2) Study in the city of Jakarta,

3) Other factors and capacity calculation formulas follow IHCM.

\section{LITERATURE REVIEW}

\section{A. Transportation Concept}

The concept of transportation is based on the trip (origin) and destination (destination). In transportation, some elements are closely related to the concept of transportation itself [1]. These elements are as follows: Humans in need, Goods needed, Vehicle's facilities, roads and terminals as transportation infrastructure, Organization (transportation manager). 


\section{B. Side Friction Factors Against Capacity Based on IHCM}

Side Friction are grouped in five classes, from very low to very high class as an accumulation of the four side obstacle factors. Classification of side Friction can be seen in TABLES I and II.

TABLE I. ClassificAtion OF Side Friction

\begin{tabular}{|l|l|l|l|}
\hline $\begin{array}{c}\text { Side } \\
\text { Friction } \\
\text { Factor } \\
\text { Level }\end{array}$ & Code & $\begin{array}{c}\text { Amount of All } \\
\text { Number of Side } \\
\text { Friction in one } \\
\text { hour }\end{array}$ & Land Use Condition \\
\hline Very Low & VL & $<100$ & $\begin{array}{l}\text { Residential area; almost } \\
\text { no activity }\end{array}$ \\
\hline Low & L & $100-299$ & $\begin{array}{l}\text { Residential area; in the } \\
\text { form of public } \\
\text { transportation and so } \\
\text { on. }\end{array}$ \\
\hline Medium & M & $300-499$ & $\begin{array}{l}\text { Commercial area; very } \\
\text { high roadside activity }\end{array}$ \\
\hline High & H & $500-899$ & $\begin{array}{l}\text { Industrial area; several } \\
\text { shops on the side of the } \\
\text { road }\end{array}$ \\
\hline Very High & VH & $>900$ & $\begin{array}{l}\text { Commercial area; } \\
\text { market activity beside } \\
\text { the road }\end{array}$ \\
\hline
\end{tabular}

TABLE II. SIDE Friction FACTORS FOR AdJUSTMENT HighWAY CAPACITY

\begin{tabular}{|c|c|c|c|c|c|}
\hline \multirow{3}{*}{$\begin{array}{c}\text { High } \\
\text { way } \\
\text { Type }\end{array}$} & \multirow{3}{*}{$\begin{array}{c}\text { Side } \\
\text { Friction } \\
\text { Factor } \\
\text { Level }\end{array}$} & \multicolumn{4}{|c|}{$\begin{array}{c}\text { Adjustment Factors for Side Barriers and } \\
\text { Kerb - Barrier Distance (FCsf) }\end{array}$} \\
\hline & & \multicolumn{4}{|c|}{ Distance Kerb-Barrier } \\
\hline & & $\leq 0,5$ & 1,0 & 1,5 & $\geq 2,0$ \\
\hline \multirow{5}{*}{$4 / 2 \mathrm{D}$} & VL & 0,95 & 0,97 & 0,99 & 1,01 \\
\hline & $\mathrm{L}$ & 0,94 & 0,96 & 0,98 & 1,00 \\
\hline & M & 0,91 & 0,93 & 0,95 & 0,98 \\
\hline & $\mathrm{H}$ & 0,86 & 0,89 & 0,92 & 0,95 \\
\hline & $\mathrm{VH}$ & 0,81 & 0,85 & 0,88 & 0,92 \\
\hline
\end{tabular}

\section{METHODOLOGY}

This research use survey data based on road observation and statistical analysis approach. Research process that explain data collection and data analysis, describe at Fig. 1.

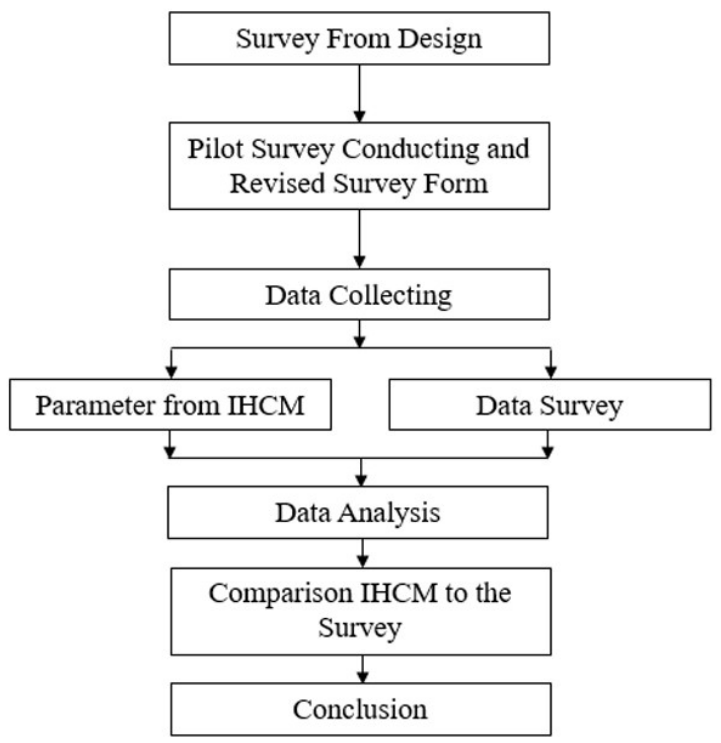

\section{VIII.DATA COLLECTION}

Data Collection method by survey on two roads in Jakarta and one road in Bogor, in Jakarta there are South Meruya road and Sacna Sunter road and in Bogor there is Kebon Pedes road. Traffic Volume analysis based on IHCM for PCU (passenger car unit motorcycle and heavy vehicle). Road was studying limited on two lanes two ways of three roads.

\section{A. Traffic Volume Survey}

Survey method with field observations carried out on the side of the road [3], the surveyor takes data on traffic volume. Time for morning data collection (06.00-08.00), daytime (11.00-13.00) and afternoon (17.00-19.00). Traffic volume data at South Meruya Jakarta, Sacna Sunter Jakarta and Kebon Pedes Bogor describe on TABLES III, IV, and V.

TABLE III. SidE Friction FACTORS FOR ADJUSTMENT HighWAY CAPACITY

\begin{tabular}{|l|l|l|l|l|}
\hline \multirow{2}{*}{$\begin{array}{c}\text { Survey } \\
\text { Time }\end{array}$} & \multicolumn{3}{|c|}{$\begin{array}{c}\text { Traffic Volume } \\
\text { (Veh/hr)) }\end{array}$} & $\begin{array}{c}\text { Q (traffic } \\
\text { volume) } \\
\text { (pcu/hour) }\end{array}$ \\
\cline { 2 - 5 } & Motor Cycle & Car & $\begin{array}{c}\text { Truk/ } \\
\text { Bus }\end{array}$ & 1289 \\
\hline $06.00-07.00$ & 3560 & 399 & 0 & 1465,75 \\
\hline $06.15-07.15$ & 4159 & 426 & 0 & 1612 \\
\hline $06.30-07.30$ & 4668 & 445 & 0 & 1720,75 \\
\hline $06.45-07.45$ & 5019 & 466 & 0 & 1660,5 \\
\hline $07.00-08.00$ & 4810 & 458 & 0 & 1536,2 \\
\hline $11.00-12.00$ & 3460 & 670 & 1 & 1634,2 \\
\hline $11.15-12.15$ & 3828 & 676 & 1 & 1611,45 \\
\hline $11.30-12.30$ & 3961 & 620 & 1 & 1562,25 \\
\hline $11.45-12.45$ & 3873 & 594 & 0 & 1496,2 \\
\hline $12.00-13.00$ & 3536 & 611 & 1 & 1720,95 \\
\hline $17.00-18.00$ & 4719 & 540 & 1 & 1556,2 \\
\hline $17.15-18.15$ & 3892 & 582 & 1 & 1435,75 \\
\hline $17.30-18.30$ & 3247 & 624 & 0 & 1366,75 \\
\hline $17.45-18.45$ & 2895 & 643 & 0 & 1306,25 \\
\hline $18.00-19.00$ & 2665 & 640 & 0 & \\
\hline
\end{tabular}

TABlE IV. ACCUMUlation TrafFic Volume SuRVEy ON SACNA SUNTER JAKARTA

\begin{tabular}{|c|l|l|l|l|}
\hline \multirow{2}{*}{$\begin{array}{c}\text { Survey } \\
\text { Time }\end{array}$} & \multicolumn{3}{|c|}{$\begin{array}{c}\text { Traffic Volume } \\
\text { (Veh/hr)) }\end{array}$} & $\begin{array}{c}\text { Q (traffic } \\
\text { volume) } \\
\text { (pcu/hour) }\end{array}$ \\
\cline { 2 - 4 } & Motor Cycle & \multicolumn{1}{|c|}{ Car } & $\begin{array}{c}\text { Truk/ } \\
\text { Bus }\end{array}$ & \\
\hline $06.00-07.00$ & 1016 & 441 & 0 & 949 \\
\hline $06.15-07.15$ & 944 & 522 & 0 & 994 \\
\hline $06.30-07.30$ & 867 & 569 & 0 & 955,5 \\
\hline $06.45-07.45$ & 919 & 559 & 0 & 1018,5 \\
\hline $07.00-08.00$ & 999 & 589 & 0 & 1088,5 \\
\hline $11.00-12.00$ & 858 & 485 & 1 & 915,3 \\
\hline $11.15-12.15$ & 965 & 495 & 0 & 977,5 \\
\hline $11.30-12.30$ & 974 & 488 & 0 & 975 \\
\hline $11.45-12.45$ & 942 & 514 & 0 & 985 \\
\hline $12.00-13.00$ & 918 & 511 & 0 & 970 \\
\hline $17.00-18.00$ & 1108 & 632 & 0 & 1186 \\
\hline $17.15-18.15$ & 1062 & 642 & 0 & 1173 \\
\hline $17.30-18.30$ & 1035 & 580 & 0 & 1097,5 \\
\hline $17.45-18.45$ & 993 & 539 & 0 & 1035,5 \\
\hline $18.00-19.00$ & 878 & 442 & 0 & 881 \\
\hline
\end{tabular}

Fig. 1. Research Flowchart 
TABLE III shows the survey result at South Meruya Jakarta shows the highest traffic volume is more than 1720 $\mathrm{pcu} /$ hour which occurs at 06.45-07.45 (morning peakhour) and 17.00-18.00 (afternoon peakhour).

TABLE IV shows the survey result at Sacna Sunter Jakarta shows the highest traffic volume is more than 1088 $\mathrm{pcu} /$ hour which occurs at 07.00-08.00 (morning peakhour) and $1097 \mathrm{pcu} /$ hour which occurs at 17.00-18.00 (afternoon peakhour).

Table V. Accumulation Traffic Volume Survey on Kebon PEDES BOGOR

\begin{tabular}{|l|l|l|l|l|}
\hline \multirow{2}{*}{$\begin{array}{c}\text { Survey } \\
\text { Time }\end{array}$} & \multicolumn{3}{|c|}{$\begin{array}{c}\text { Traffic Volume } \\
\text { (Veh/hr) }\end{array}$} & \multicolumn{1}{c|}{$\begin{array}{c}\text { Q (traffic } \\
\text { volume) } \\
\text { (pcu/hour) }\end{array}$} \\
\cline { 2 - 4 } & Motor Cycle & Car & $\begin{array}{c}\text { Truk/ } \\
\text { Bus }\end{array}$ & \multicolumn{1}{|c|}{} \\
\hline $06.00-07.00$ & 3190 & 430 & 0 & 1227,5 \\
\hline $06.15-07.15$ & 3526 & 435 & 0 & 1316,5 \\
\hline $06.30-07.30$ & 3655 & 411 & 0 & 1324,75 \\
\hline $06.45-07.45$ & 3555 & 354 & 0 & 1242,75 \\
\hline $07.00-08.00$ & 3382 & 328 & 0 & 1173,5 \\
\hline $11.00-12.00$ & 2123 & 480 & 1 & 1010,75 \\
\hline $11.15-12.15$ & 2118 & 473 & 1 & 1002,5 \\
\hline $11.30-12.30$ & 2112 & 485 & 0 & 1013 \\
\hline $11.45-12.45$ & 2086 & 507 & 0 & 1028,5 \\
\hline $12.00-13.00$ & 2152 & 504 & 0 & 1042 \\
\hline $17.00-18.00$ & 2520 & 308 & 0 & 938 \\
\hline $17.15-18.15$ & 2107 & 308 & 0 & 834,75 \\
\hline $17.30-18.30$ & 1756 & 297 & 0 & 736 \\
\hline $17.45-18.45$ & 1527 & 278 & 0 & 659,75 \\
\hline $18.00-19.00$ & 1600 & 279 & 0 & 679 \\
\hline
\end{tabular}

TABLE V shows the survey result at Kebon Pedes Bogor shows the highest traffic volume is more than $1324 \mathrm{pcu} /$ hour which occurs at 06.30-07.30 (morning peakhour) and theres is not afternoon peakhour.

Based on survey the condition or data of side Friction at three roads that survey conducted describe at TABLES VI, VII, VIII.

TABle VI. Side Friction Data at South Meruya Road JaKarta

\begin{tabular}{|c|c|c|c|c|}
\hline \multirow[b]{2}{*}{ Time Slice } & \multicolumn{4}{|c|}{ Side Friction } \\
\hline & $\begin{array}{c}\text { Un } \\
\text { Motorised } \\
\text { Vehicle } \\
\end{array}$ & $\begin{array}{c}\text { Pedest } \\
\text { rian }\end{array}$ & Access & $\begin{array}{l}\text { Parking } \\
\text { Vehicle }\end{array}$ \\
\hline $06.00-07.00$ & 9 & 21 & 828 & 25 \\
\hline $06.15-07.15$ & 14 & 21 & 1226 & 33 \\
\hline $06.30-07.30$ & 13 & 53 & 1809 & 51 \\
\hline $06.45-07.45$ & 15 & 86 & 2312 & 64 \\
\hline $07.00-08.00$ & 15 & 102 & 2654 & 68 \\
\hline $11.00-12.00$ & 5 & 108 & 1251 & 38 \\
\hline $11.15-12.15$ & 5 & 120 & 1170 & 39 \\
\hline $11.30-12.30$ & 3 & 120 & 1245 & 38 \\
\hline $11.45-12.45$ & 3 & 109 & 1263 & 45 \\
\hline $12.00-13.00$ & 4 & 113 & 1190 & 51 \\
\hline $17.00-18.00$ & 11 & 97 & 2506 & 56 \\
\hline $17.15-18.15$ & 13 & 80 & 2085 & 40 \\
\hline $17.30-18.30$ & 10 & 68 & 1736 & 31 \\
\hline $17.45-18.45$ & 7 & 59 & 1395 & 20 \\
\hline $18.00-19.00$ & 5 & 51 & 1133 & 13 \\
\hline
\end{tabular}

TABLE VI shows the survey result at South Meruya Jakarta shows the highest side friction is access (2654 vehicle) which occurs at 07.00-08.00 AM.

TABLE VII. Side Friction Data AT SACNA Sunter RoAd JAKARTA

\begin{tabular}{|c|c|c|c|c|}
\hline \multirow[b]{2}{*}{ Time Slice } & \multicolumn{4}{|c|}{ Side Friction } \\
\hline & $\begin{array}{c}\text { Un } \\
\text { Motorised } \\
\text { Vehicle }\end{array}$ & $\begin{array}{c}\text { Pedest } \\
\text { rian }\end{array}$ & Access & $\begin{array}{l}\text { Parking } \\
\text { Vehicle }\end{array}$ \\
\hline $06.00-07.00$ & 11 & 13 & 424 & 5 \\
\hline $06.15-07.15$ & 11 & 12 & 430 & 3 \\
\hline $06.30-07.30$ & 12 & 11 & 440 & 1 \\
\hline $06.45-07.45$ & 11 & 14 & 454 & 3 \\
\hline $07.00-08.00$ & 16 & 20 & 456 & 5 \\
\hline $11.00-12.00$ & 10 & 16 & 410 & 16 \\
\hline $11.15-12.15$ & 12 & 17 & 441 & 16 \\
\hline $11.30-12.30$ & 13 & 18 & 492 & 17 \\
\hline $11.45-12.45$ & 16 & 18 & 521 & 15 \\
\hline $12.00-13.00$ & 20 & 18 & 516 & 8 \\
\hline $17.00-18.00$ & 21 & 32 & 460 & 10 \\
\hline $17.15-18.15$ & 13 & 28 & 442 & 6 \\
\hline $17.30-18.30$ & 9 & 19 & 440 & 3 \\
\hline $17.45-18.45$ & 10 & 11 & 445 & 1 \\
\hline $18.00-19.00$ & 6 & 14 & 425 & 3 \\
\hline
\end{tabular}

TABLE VII shows the survey result at Sacna Sunter Jakarta shows the highest side friction is access (492 vehicle) which occurs at 11.30-12.30 AM.

TABle ViII. Side Friction Data at Kebon Pedes Road Bogor

\begin{tabular}{|l|l|l|l|l|}
\hline \multirow{2}{*}{ Time Slice } & \multicolumn{4}{|c|}{ Side Friction } \\
\cline { 2 - 5 } & $\begin{array}{c}\text { Un } \\
\text { Motorised } \\
\text { Vehicle }\end{array}$ & $\begin{array}{c}\text { Pedest } \\
\text { rian }\end{array}$ & Access & $\begin{array}{c}\text { Parking } \\
\text { Vehicle }\end{array}$ \\
\hline $06.00-07.00$ & 4 & 90 & 326 & 120 \\
\hline $06.15-07.15$ & 6 & 101 & 367 & 140 \\
\hline $06.30-07.30$ & 6 & 96 & 382 & 143 \\
\hline $06.45-07.45$ & 4 & 100 & 340 & 121 \\
\hline $07.00-08.00$ & 3 & 95 & 279 & 98 \\
\hline $11.00-12.00$ & 6 & 76 & 282 & 67 \\
\hline $11.15-12.15$ & 3 & 87 & 298 & 78 \\
\hline $11.30-12.30$ & 0 & 98 & 313 & 79 \\
\hline $11.45-12.45$ & 0 & 95 & 326 & 82 \\
\hline $12.00-13.00$ & 0 & 93 & 337 & 91 \\
\hline $17.00-18.00$ & 9 & 48 & 269 & 50 \\
\hline $17.15-18.15$ & 9 & 38 & 250 & 45 \\
\hline $17.30-18.30$ & 5 & 32 & 208 & 40 \\
\hline $17.45-18.45$ & 2 & 32 & 215 & 40 \\
\hline $18.00-19.00$ & 1 & 43 & 232 & 37 \\
\hline & & & & \\
\hline
\end{tabular}

TABLE VIII shows the survey result at Kebon Pedes Bogor shows the highest side friction is access (382 vehicle) which occurs at 06.30-07.30 AM.

\section{DATA ANALYSIS}

To see the relationship between traffic volume and side friction, comparison value of each weighted factor between IHCM with survey analysis show at TABLE IX. 
TABLE IX shows the position of each side friction factor at IHCM and correlation between traffic volume and survey data analysis is appropriate. The differencies betweeen IHCM and survey data analysis for side friction factor describe at TABLE $X$.

TABLE IX. RELATIONSHIP OF TRAFFic Volume With SidE Friction

\begin{tabular}{|l|l|l|l|l|}
\hline \multirow{2}{*}{$\begin{array}{c}\text { Side } \\
\text { Friction } \\
\text { Factor }\end{array}$} & \multirow{2}{*}{ IHCM } & \multicolumn{3}{|c|}{ Pearson Correlation (R) } \\
\cline { 3 - 5 } & $\begin{array}{c}\text { Meruya } \\
\text { Selatan,Jkt }\end{array}$ & $\begin{array}{c}\text { Sacna } \\
\text { Sunter,Jkt }\end{array}$ & $\begin{array}{c}\text { Kb.Pedas } \\
\text { Bogor }\end{array}$ \\
\hline $\begin{array}{l}\text { Un } \\
\text { Motorized } \\
\text { Vehicle }\end{array}$ & 0,4 & 0,3 & 0,4 & 0,2 \\
\hline Pedestrian & 0,5 & 0,6 & 07 & 0,8 \\
\hline $\begin{array}{l}\text { Left-Right } \\
\text { Acces }\end{array}$ & 0,7 & 0,7 & 0,7 & 0,9 \\
\hline $\begin{array}{l}\text { Parked/ } \\
\text { Stopped } \\
\text { Vehicle }\end{array}$ & 1 & 0,9 & 0,8 & 1 \\
\hline
\end{tabular}

TABlE X. Percentage Differencies Between Survey

\begin{tabular}{|l|l|l|l|l|l|l|}
\hline \multicolumn{1}{|c|}{ Road } & \multicolumn{1}{|c|}{ Description } & $\begin{array}{c}\text { Total Number } \\
\text { Side Friction }\end{array}$ & $\begin{array}{c}\text { Level } \\
\text { Classification }\end{array}$ & \multicolumn{1}{|c|}{ FCsf } & \multicolumn{1}{c|}{ Percentage VH Level } & $\begin{array}{c}\text { Percentage M } \\
\text { Level }\end{array}$ \\
\hline Meruya Selatan & Campus, Restaurant & $1000-2000$ & VH & 0,72 & $2 \%$ & - \\
\hline Sacna Sunter & Shop & $300-400$ & M & 0,88 & - & $4 \%$ \\
\hline Kb.Pedas Bogor & School, Shop & $300-450$ & $\mathrm{M}$ & 0,88 & - & $10 \%$ \\
\hline
\end{tabular}

TABLE X based on RMSE (Root Mean Square Error) the differencies of each side friction factor based on IHCM and survey data analysis is very close at Very High level classification or about $2 \%$, and for Medium level classification is about at the range $4 \%-10 \%$.

\section{CONCLUSIOnS}

a) Weight Sequence in IHCM for side friction more close with data analysis for $\mathrm{VH}$, but rather difference at Medium Level Side Friction.

b) The value of weight side friction in IHCM and data analysis is more difference at urban with nondensity population.

c) In the survey land use of very high and medium road side activity is appropriate with categorized in IHCM.

\section{REFERENCES}

[1] Tamin, O Z, Transportation Planning and Modelling, Bandung: Publisher ITB, 2000.

[2] IHCM, Indonesia Highway Capacity Manual, Directorate General of Highways, Ministry of Public Works Indonesia, 1997.

[3] Lowinta L, Najid, Evaluation and Comparison Road Capacity Based on IHCM, PKJI and Survey at Road Two Lanes Two Ways, Civil Engineering Journal, Tarumanagara University, 2019 